\title{
Análise das Ações de Vigilância do Desenvolvimento Infantil Segundo Cuidadores de Crianças
}

\section{Analysis of Child Development Surveillance Actions According to Child Caregivers}

\author{
Iolanda Carlli da Silva Bezerra ${ }^{1}$
}

Tayná Lima dos Santos ${ }^{2}$

Amanda Evelyn Valença de Melo $^{3}$

Daniele Beltrão Lucena de França ${ }^{4}$

Daniele de Souza Vieira ${ }^{5}$

Tarciane Marinho Albuquerque de Vasconcellos $\mathrm{Cruz}^{6}$

Altamira Pereira da Silva Reichert ${ }^{7}$

\section{RESUMO}

Objetivo: Analisar as ações de vigilância do desenvolvimento infantil realizadas por profissionais de saúde, segundo cuidadores de crianças. Metodologia: Estudo transversal, realizado em Unidades de Saúde da Família de João Pessoa-PB, no período de maio a julho de 2019. Participaram da pesquisa 145 mães e/ou cuidadores de crianças menores de três anos de idade. Para análise dos dados, utilizou-se estatística descritiva e inferencial, com o teste de Fisher. Resultados: Segundo os cuidadores $(60,1 \%)$, os profissionais que atenderam a criança não perguntaram a sua opinião acerca do desenvolvimento das crianças; não foram orientados a como estimular a criança no domicílio $(55,2 \%)$ e não receberam informações sobre a Caderneta de Saúde da Criança $(57,4$ \%). Ocoreu associação significativa entre as variáveis percepção do desenvolvimento com a renda, orientação sobre como estimular a criança no domicílio, com as variáveis número de filhos e renda e entre as variáveis número de filhos e renda, com o fato do profissional mostrar o registro ao cuidador. Conclusão: Os profissionais de saúde não estão realizando a vigilância do desenvolvimento infantil conforme preconizado, como também, não estão incluindo a família como colaboradora da vigilância do desenvolvimento na Caderneta da Criança, fato este que dificulta a continuidade do seguimento da criança na Atenção Primária à Saúde e a promoção do desenvolvimento saudável.

\section{DESCRITORES}

Saúde da Criança. Desenvolvimento Infantil. Atenção Primária à Saúde. Pais. Trabalhadores da Saúde.

\begin{abstract}
Objective: To analyze the child development surveillance actions carried out by health professionals, according to child caregivers. Methodology: Cross-sectional study, carried out in Family Health Units in João Pessoa-PB, from May to July 2019. 145 mothers and/or caregivers of children under three years of age participated in the research. For data analysis, descriptive and inferential statistics were used, with Fisher's test. Results: According to caregivers $(60.1 \%)$, the professionals who assisted the child did not ask their opinion about the children's development; they were not instructed on how to stimulate the child at home $(55.2 \%)$ and did not receive information on the Child Health Handbook (57.4\%). There was a significant association between the variables perception of development with income, guidance on how to stimulate the child at home, with the variables number of children and income, and between the variables number of children and income with the fact that the professional shows the record to the child's caregiver. Conclusion: Health professionals are not conducting child development surveillance as recommended, nor are they including the family as a development surveillance collaborator in the Caderneta da Criança, a fact that hinders the continuity of child follow-up in Primary Health Care and promotes healthy development.
\end{abstract}

\section{DESCRIPTORS}

Child Health. Child Development. Primary Health Care. Parents. Health Workers.

${ }^{1}$ Acadêmica de Enfermagem pela Universidade Federal da Paraíba. João Pessoa, Paraíba, Brasil. ORCID iD: https://orcid.org/00000002-7948-8074.

${ }^{2}$ Acadêmica de Enfermagem pela Universidade Federal da Paraíba. João Pessoa, Paraíba, Brasil. ORCID iD: https://orcid.org/00000002-3472-0992.

${ }^{3}$ Acadêmica de Medicina pela Universidade Federal da Paraíba. João Pessoa, Paraíba, Brasil. ORCID iD: https://orcid.org/0000-00022674-031X.

${ }^{4}$ Enfermeira. Mestranda do Programa de Pós-Graduação em Enfermagem da Universidade Federal da Paraíba. João Pessoa, Paraíba, Brasil.ORCID iD: https://orcid.org/0000-0003-1886-0474.

${ }^{5}$ Enfermeira. Doutoranda do Programa de Pós-Graduação em Enfermagem da Universidade Federal da Paraíba. João Pessoa, Paraíba, Brasil. ORCID iD: https://orcid.org/0000-0002-5747-9513.

${ }^{6}$ Enfermeira da Estratégia Saúde da Família do Município de João Pessoa- PB, Doutoranda pelo Programa de Pós-Graduação em Enfermagem da Universidade Federal da Paraíba, ORCID iD: https://orcid.org/0000-0003-0780-117X.

${ }^{7}$ Enfermeira. Doutora em Saúde da Criança e Adolescente. Docente do Programa de Pós-Graduação em Enfermagem da Universidade Federal da Paraíba. João Pessoa, Paraíba, Brasil. ORCID iD: http://orcid.org/0000-0002-4295-6698. 
A primeira infância apresenta importância singular no desenvolvimento humano, pois é nessa fase que ocorrem eventos cruciais no crescimento e no desenvolvimento infantil. Crianças que possuem uma infância desfavorecida em aspectos econômicos e sociais correm maior risco de não alcançarem seu potencial de desenvolvimento ${ }^{1,2}$.

Assim, são necessários investimentos em ações que priorizem a promoção da saúde nos primeiros anos de vida, pois evidências apontam para a importância da assistência nesse período, no qual existe a possibilidade de maximizar o potencial de desenvolvimento infantil. Contrariamente, falhas em tais investimentos acarretam diversas implicações negativas para as crianças, suas famílias e para a sociedade ${ }^{2,3}$.

Todavia, mesmo diante de avanços significativos em pesquisas, programas e políticas para o desenvolvimento infantil no mundo, cerca de 250 milhões de crianças que vivem em países com média e baixa renda estão em risco para o desenvolvimento ${ }^{4}$. Em João Pessoa, Capital da Paraíba - Brasil, uma pesquisa evidenciou uma prevalência de $52,7 \%$ de alteração no desenvolvimento de crianças de seis a dezoito meses de vida ${ }^{5}$.

Diante desse contexto ressalta-se a importância de um seguimento adequado na primeira infância, podendo ser concretizado por meio da Vigilância do Desenvolvimento Infantil (VDI) que objetiva promover o desenvolvimento normal e detectar problemas e disfunções nos primeiros anos de vida, viabilizando a prevenção de enfermidades e resolutividade dessas em tempo oportuno para evitar complicações ${ }^{6}$.
No Brasil, a Política Nacional de Atenção Integral à Saúde da Criança (PNAISC) objetiva promover a integralidade na assistência por meio de sete eixos estratégicos, dentre eles, a promoção e o acompanhamento do crescimento e o desenvolvimento integral. Esta política recomenda que as ações estratégicas propostas para cada eixo sejam implementadas na Rede de Atenção à Saúde (RAS), tendo a Atenção Primária à Saúde (APS) como coordenadora do cuidado ${ }^{7}$.

De acordo com as diretrizes de atenção à saúde da criança, a VDI contempla ações relacionadas à avaliação dos marcos do desenvolvimento infantil; verificação da presença de fatores de risco; busca de opinião das mães sobre o desenvolvimento do seu fiIho; verificação da maneira como a mãe/cuidador e familiares, em geral, relacionam-se com a criança e, por fim, realização dos registros e interpretação dos achados na Caderneta de Saúde da Criança (CSC).

A CSC é uma importante ferramenta de vigilância do crescimento e desenvolvimento na infância tendo em vista que viabiliza o seguimento desde o nascimento até os dez anos de idade ${ }^{2,9}$. Os registros contidos na caderneta são essenciais para a realização do acompanhamento infantil, uma vez que, por meio deles, é possível identificar agravos, intercorrências e informações acerca da saúde e do desenvolvimento na infância, além de promoverem a comunicação entre profissionais de saúde e familiares da criança ${ }^{10,11}$.

No entanto, alguns profissionais de saúde não reconhecem a família como núcleo participativo do cuidado à criança, no que concerne ao preenchimento de dados na CSC, confiando o registro dos dados apenas 
a equipe de saúde ${ }^{10}$, embora, empiricamente, saiba-se que essa é uma ação que pode ser comumente conversada e também compartiIhada com os responsáveis pela criança. Logo, a participação familiar, tanto no que se refere ao preenchimento, quanto no acompanhamento dos registros realizados na caderneta é de suma relevância, visto que a família é grande aliada na efetivação da VDI ${ }^{6,10,12}$.

Pesquisas brasileiras e internacionais evidenciam ainda a importância e o potencial da família na estimulação do desenvolvimento e na promoção da VDI e ainda revelam que o envolvimento da família, com apoio da atenção primária, tem capacidade de fomentar o desenvolvimento neurológico das crianças na primeira infância ${ }^{6,12,13}$. Entretanto, estudos apontam para a falta de qualquer tipo de anotação das mães nas cadernetas da criança, como também, a falta de orientações dos profissionais quanto à estimulação do desenvolvimento da criança no ambiente domiciliar ${ }^{14,15}$.

Uma das atribuições dos profissionais de saúde na VDI é orientar a família e envolvê-la na estimulação do desenvolvimento e na identificação dos sinais de risco para saúde da criança. Ademais, ao atuar nas consultas de acompanhamento das crianças, os profissionais avaliam o estado de saúde, o que possibilita a intervenção precoce de acordo com as necessidades da criança ${ }^{2,7,10,15}$.

Diante do exposto, surgiram os seguintes questionamentos: Quais as ações de VDI são realizadas pelos profissionais de saúde? De que forma a família é envolvida pelos profissionais no processo de VDI?

Dessa forma, ante a relevância do núcleo familiar no desenvolvimento da criança e por reconhecer que os pais exercem um papel fundamental na estimulação do filho e da falta de envolvimento da família na Vigilância do Desenvolvimento Infantil pelos profissionais de saúde, o que acarreta em lacuna no acompanhamento do crescimento e desenvolvimento, este estudo tem como objetivo analisar as ações de vigilância do desenvolvimento infantil realizadas por profissionais de saúde, segundo cuidadores de crianças. Esse estudo se justifica por considerar que são necessárias decisões assertivas que envolvam os familiares das crianças na promoção do seu desenvolvimento, uma vez que as alterações no desenvolvimento infantil geram agravos à saúde e afetam todo o seu contexto de vida.

\section{METODOLOGIA}

Estudo transversal, realizado em Unidades de Saúde da Família (USF) do Distrito Sanitário I de João Pessoa - Paraíba. O município dispõe de uma rede de atenção básica organizada territorialmente a partir de cinco Distritos Sanitários (DS), que coordenam 200 Equipes de Saúde da Família (EqSF). A escolha pelo DS I se deu por meio de um sorteio aleatório entre os cinco distritos de João Pessoa.

O DS I é responsável pela cobertura de $90,5 \%$ da população adscrita, possuindo 50 EqSF. No período em que a pesquisa foi realizada, contava com 729 crianças menores de seis meses e 6.183 crianças menores de cinco anos de idade.

Os participantes da pesquisa foram mães e/ou cuidadores de crianças menores de três anos de idade, cadastradas nas USF do Distrito Sanitário I, maiores de 18 anos, 
que portavam a CSC no momento da coleta de dados e que eram atendidas nesses serviços de saúde. Excluiu-se do estudo aqueles que apresentaram limitações consideráveis quanto ao entendimento dos questionamentos e capacidade de resposta, tais como, incapacidade de compreensão das perguntas e de leitura, compreensão do termo de consentimento livre e esclarecido (TCLE).

Para o cálculo amostral considerou-se o número de nascidos vivos dos anos de 2016, 2017 e 2018, totalizando a população de 4.102 crianças de zero a três anos que estavam sob a tutela de um cuidador legal. A seleção da amostra foi realizada pelo método de alocação ótima, considerando a população total, ao final, chegou-se um valor estimado de 116 cadernetas de crianças e mães/ cuidadores. No entanto, foi acrescentado um percentual de $20 \%$ de eventuais perdas, constituindo assim, uma amostra final de 145 mães/cuidadores respondentes. Esse quantitativo foi distribuído de acordo com o número de crianças cadastradas em cada unidade de saúde do referido Distrito, sendo escolhidas para a coleta de dados àquelas que possuíam maior quantitativo de crianças cadastradas.

Os dados foram coletados no período de maio a julho de 2019. A seleção dos participantes nas unidades de saúde foi por conveniência ${ }^{16}$, na fila de espera para consulta de puericultura ou atendimento na sala de vacina. A última mãe e/ou cuidador legal da criança presente na fila era convidada a participar da pesquisa e, quando esta não aceitava participar, o anterior que se encontrava na fila era convidado, e assim sucessivamente, até se completar o quantitativo amostral estabelecido para cada USF.
A pesquisa seguiu as recomendações éticas de estudos com seres humanos, sendo entregue o Termo de Consentimento Livre e Esclarecido (TCLE) e somente após a leitura e consentimento do participante é que se procedeu a coleta. Destaca-se que todos os participantes receberam uma cópia do TCLE.

As entrevistas foram norteadas por um questionário do tipo check-list, tendo com embasamento teórico a CSC e as recomendações do Ministério da Saúde. O instrumento contemplou na primeira parte os dados de identificação da USF; na segunda parte, informações acerca das condições demográficas e socioeconômicas do cuidador da criança, além da característica da criança; e uma terceira parte com perguntas referentes ao envolvimento dos cuidadores na Vigilância do Desenvolvimento Infantil e a utilização da CSC durante a consulta, por parte dos profissionais que atenderam à criança.

Os dados foram tabulados e digitados em dupla entrada para verificação de inconsistências e correção da digitação para, posteriormente, iniciar o processo da análise estatística descritiva com cálculo da frequência absoluta e relativa bem como aplicação do Teste de Fisher para verificação de associação entre variáveis, com base em um nível de confiança de $95 \%$. Apesar de ser empregado na prática para estudos com amostras pequenas, o teste exato de Fisher é válido para todos os tamanhos amostrais ${ }^{16}$.

Este estudo é vinculado ao projeto universal "Vigilância do desenvolvimento e a caderneta de saúde da criança: caminhos para a promoção da saúde infantil" número 407850/2018-0 e aprovado pelo comitê de ética do Centro de do Centro de Ciências da 
Saúde da Universidade Federal da Paraíba, com CAAE 97362718.1.0000.5188.

\section{RESULTADOS}

A Tabela 1 apresenta as características sociodemográficas dos participantes na qual se observa que $77,9 \%$ compreendiam a faixa etária entre 29 a 39 anos, $44,8 \%$ afirmaram estar em união estável, $40,0 \%$ relataram possuir dois filhos vivos, $71,7 \%$ declararam não estarem trabalhando e $82,4 \%$ tinham mais de oito anos de estudo. No que se refere à quantidade de pessoas residentes no mesmo domicílio, 53,1\% conviviam com quatro ou cinco pessoas e $69,0 \%$ declararam existir apenas uma criança menor de cinco anos. A renda familiar predominante foi inferior ao salário mínimo vigente. Quanto às crianças, $39,3 \%$ tinha entre 12 e 23 meses de idade, com predominância do sexo feminino $(52,4 \%)$.

Com relação às perguntas relacionadas ao envolvimento dos cuidadores na vigilância do desenvolvimento infantil e a utilização da CSC durante a consulta realizada pelos profissionais, conforme a Tabela 2 evidencia-se que $60,1 \%$ de mães e/ou cuidadores responderam que não foram questionados pelos profissionais acerca da sua percepção em relação ao desenvolvimento da criança. Cerca de 59,0\% afirmaram receber informações sobre o desenvolvimento da criança durante a consulta, entretanto, a maioria $(55,2 \%)$ reiterou não ter recebido nenhuma informação/orientação sobre como estimular a criança no domicílio. Quanto à utilização da CSC, $57,4 \%$ responderam que não receberam informações sobre a CSC pelos profissionais de saúde e $52,4 \%$ informaram que os pro- fissionais costumavam mostrar os registros presentes na caderneta da criança.

No que tange a análise da associação entre as variáveis sobre o envolvimento dos cuidadores na vigilância do desenvolvimento infantil durante a consulta à criança por parte dos profissionais, a partir da visão das mães e/ou cuidadores, com alguns dados sociodemográficos e econômicos, a Tabela 3 revela significância estatística apenas entre as variáveis: o profissional perguntou sua percepção acerca do desenvolvimento, com a renda familiar; se a mãe/cuidadora recebeu orientação quanto à estimulação da criança no domicílio, com as variáveis número de filhos e renda familiar. Em relação à pergunta se o profissional mostrou o registro da CSC ao cuidador, nota-se significância também com as variáveis número de filhos e renda familiar.

\section{DISCUSSÃO}

A família tem papel fundamental na Vigilância do Desenvolvimento Infantil. Mesmo em contextos de vulnerabilidades, os recursos familiares têm capacidade de intervir positivamente no potencial infantil, levando as crianças a alcançarem um desenvolvimento ótimo ${ }^{13}$. Em vista disso, o momento da puericultura é bastante oportuno para fornecer orientações acerca do desenvolvimento e da estimulação domiciliar aos responsáveis pela criança ${ }^{15,17}$. No entanto, os dados desta pesquisa evidenciam que os cuidadores não estão sendo incluídos no processo da VDI pelos profissionais de saúde da Atenção Básica $(A B)$, fato este que pode prejudicar o desenvolvimento na primeira infância. 
Tabela 1. Distribuição das características sociodemográficas e econômicas de cuidadores e características das crianças, João Pessoa, PB, Brasil, 2019.

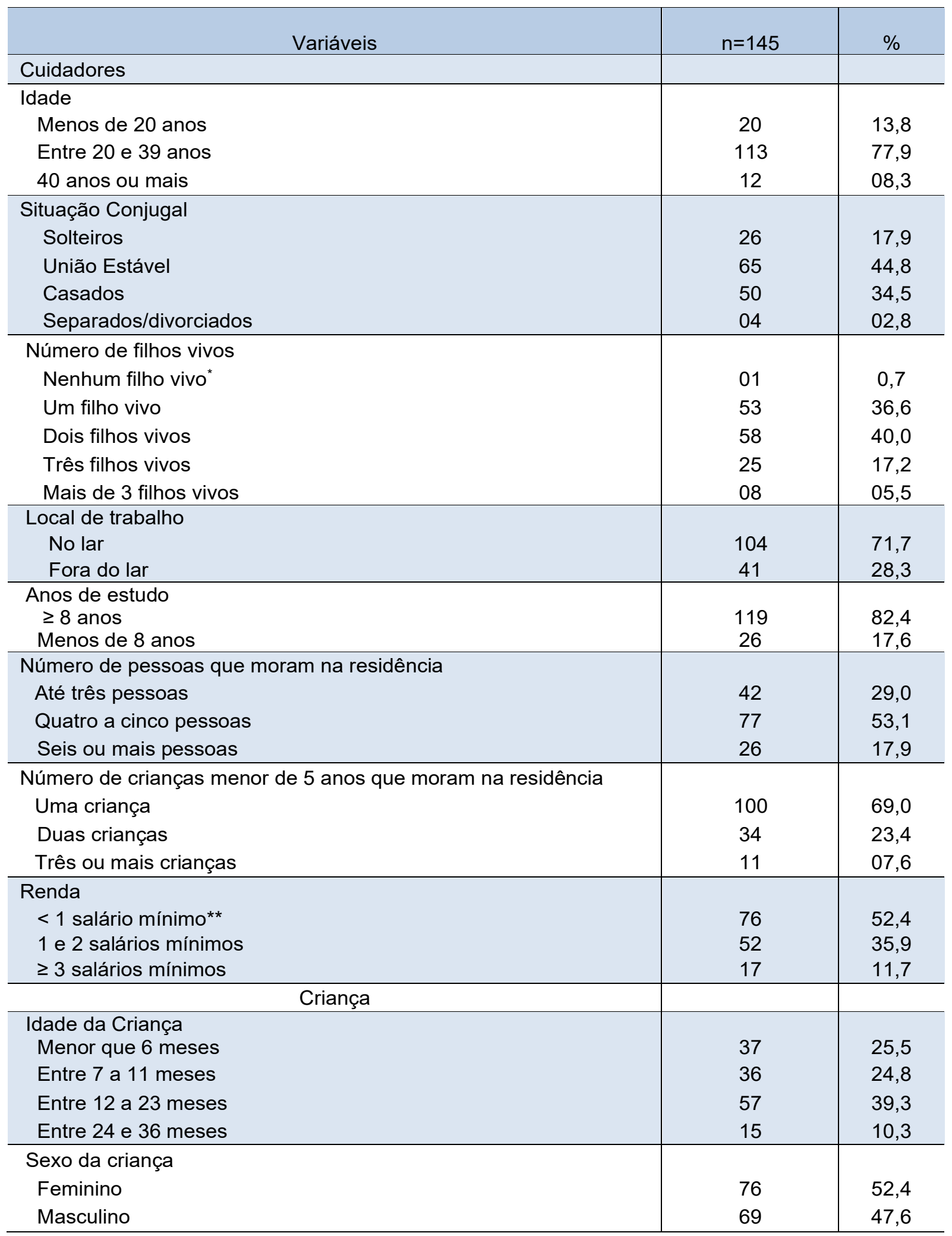

* A cuidadora era a avó da criança. ** Salário mínimo vigente de $\mathrm{R} \$$ 998,00 em janeiro de 2019. 
Tabela 2. Informações dos cuidadores acerca da conduta profissional na vigilância do desenvolvimento na consulta à criança. João Pessoa, PB, Brasil, 2019.

\begin{tabular}{l|c|c}
\hline \multicolumn{1}{c|}{ Vigilância do Desenvolvimento } & $\mathrm{n}=145$ & $\%$ \\
\hline Profissional perguntou a opinião sobre o desenvolvimento da criança & 57 & 39,2 \\
Sim & 87 & 60,1 \\
Não & 01 & 0,7 \\
Não se lembra & & \\
\hline Recebeu informação acerca do desenvolvimento da criança & 86 & 59,4 \\
Sim & 58 & 39,9 \\
Não & 01 & 0,7 \\
Não se lembra & & \\
\hline Recebeu orientação quanto à estimulação domiciliar & 64 & 44,1 \\
Sim & 80 & 55,2 \\
Não & 01 & 0,7 \\
Não se lembra & & \\
\hline Recebeu informação sobre a CSC & 59 & 40,7 \\
Sim & 83 & 57,4 \\
Não & 03 & 2,1 \\
Não se lembra & & \\
\hline O profissional mostrou o registro na caderneta & 76 & 52,4 \\
Sim & 65 & 44,8 \\
Não & 04 & 2,8 \\
Não se lembra &
\end{tabular}

A maioria das mães e/ou cuidadores afirmaram que os profissionais médicos e/ou enfermeiros não costumam perguntar a sua opinião acerca do desenvolvimento dos seus filhos, semelhante a estudos internacionais que também apontaram falha do profissional nesse aspecto ${ }^{18,19}$. Contrariamente, estudo de intervenção com profissionais da Estratégia Saúde da Família para capacitação em Vigilância do Desenvolvimento Infantil, evidenciou que $73 \%$ desses costumavam perguntar a opinião das mães sobre o desenvolvimento de seus filhos e, após a intervenção, todos passaram a perguntar ${ }^{6}$, evidenciando a importância da qualificação profissional para a vigilância do desenvolvimento infantil ${ }^{10,11}$.

Outro dado que chama atenção é que mais da metade dos cuidadores afirmaram não receber orientação de como estimular a criança no domicílio, igualmente a pesquisa realizada no Mato Grosso, com enfermeiros de USFs ${ }^{15}$. É fundamental que os cuidadores sejam orientados quanto a estimulação infantil no domicílio, tendo em vista que o ambiente doméstico afeta os diferentes domínios do desenvolvimento na primeira infância, conforme constatou estudo de coorte com 502 pares mãe-filho, na Itália ${ }^{20}$.

Ademais, os dados apontam para faIhas na Vigilância do Desenvolvimento Infantil, uma vez que os profissionais não envolvem a família na consulta, fato esse que dificulta a autonomia familiar para a promoção do crescimento e do desenvolvimento saudável. Nesse contexto, é fundamental que os profissionais de saúde atuem de modo a fortalecer os vín- 
Tabela 3. Associação significativa entre o envolvimento dos cuidadores acerca da vigilância do desenvolvimento na consulta à criança, realizadas pelos profissionais com os dados sociodemográficos e econômicos. João Pessoa, PB, Brasil, 2019.

\begin{tabular}{|c|c|c|c|c|}
\hline \multirow[t]{2}{*}{ Variáveis } & \multicolumn{4}{|c|}{$\begin{array}{c}\text { O profissional pergunta a opinião sobre desenvolvimento da } \\
\text { criança } \\
n n=145\end{array}$} \\
\hline & $\operatorname{Sim}(\%)$ & Não (\%) & Não se lembra (\%) & $\mathrm{p}$-valo \\
\hline $\begin{array}{l}\text { Renda } \\
<1 \text { salário mínimo } \\
1 \text { a } 2 \text { salários mínimos } \\
3 \text { ou mais salários mínimos }\end{array}$ & $\begin{array}{c}27(18,6) \\
19(13,1) \\
10(6,9)\end{array}$ & $\begin{array}{c}48(33,1) \\
32(22,1) \\
06(4,1)\end{array}$ & $\begin{array}{l}01(0,7) \\
01(0,7) \\
01(0,7)\end{array}$ & 0,05 \\
\hline \multirow{2}{*}{3 ou mais salários mínimos } & \multicolumn{4}{|c|}{ Recebeu orientação quanto à estimulação domiciliar } \\
\hline & $\operatorname{Sim}(\%)$ & Não (\%) & Não se lembra (\%) & p-valo \\
\hline $\begin{array}{l}\text { Número de filhos vivos } \\
\text { Nenhum filho vivo } \\
\text { Um filho vivo } \\
\text { Dois filhos vivos } \\
\text { Três filhos vivos } \\
\text { Mais de } 3 \text { filhos vivos }\end{array}$ & $\begin{array}{c}- \\
19(13,1) \\
29(20,0) \\
12(8,3) \\
03(2,1)\end{array}$ & $\begin{array}{c}- \\
34(23,4) \\
29(20,0) \\
11(7,6) \\
05(3,4)\end{array}$ & $\begin{array}{c}01(0,7) \\
- \\
- \\
02(1,4) \\
-\end{array}$ & 0,031 \\
\hline $\begin{array}{l}\text { Renda } \\
<1 \text { salário mínimo } \\
1 \text { a } 2 \text { salários mínimos } \\
3 \text { ou mais salários mínimos }\end{array}$ & $\begin{array}{c}28(19,3) \\
24(16,6) \\
11(7,6)\end{array}$ & $\begin{array}{c}47(32,4) \\
27(18,6) \\
05(3,4)\end{array}$ & $\begin{array}{l}01(0,7) \\
01(0,7) \\
01(0,7)\end{array}$ & 0,024 \\
\hline \multirow{2}{*}{3 ou mais salários mínimos } & \multicolumn{4}{|c|}{ O profissional mostrou o registro na caderneta } \\
\hline & $\operatorname{Sim}(\%)$ & Não $(\%)$ & Não se lembra (\%) & p-valo \\
\hline $\begin{array}{l}\text { Número de filhos vivos } \\
\text { Nenhum filho vivo } \\
\text { Um filho vivo } \\
\text { Dois filhos vivos } \\
\text { Três filhos vivos } \\
\text { Mais de } 3 \text { filhos vivos }\end{array}$ & $\begin{array}{c}- \\
30(20,7) \\
30(20,7) \\
14(9,6) \\
02(1,4)\end{array}$ & $\begin{array}{c}- \\
22(15,2) \\
28(19,3) \\
10(6,9) \\
05(3,4)\end{array}$ & $\begin{array}{l}01(0,7) \\
01(0,7) \\
- \\
01(0,7) \\
01(0,7)\end{array}$ & 0,032 \\
\hline $\begin{array}{l}\text { Renda } \\
<1 \text { salario mínimo } \\
1 \text { a } 2 \text { salários mínimos } \\
3 \text { ou mais salários mínimos }\end{array}$ & $\begin{array}{c}32(22,1) \\
31(21,4) \\
13(8,9)\end{array}$ & $\begin{array}{c}42(28,9) \\
21(14,5) \\
02(1,4)\end{array}$ & $\begin{array}{l}02(1,4) \\
- \\
02(1,4)\end{array}$ & 0,001 \\
\hline
\end{tabular}

culos familiares para a estimulação precoce e apropriada, a fim de garantir que todas as crianças alcancem seu pleno potencial de desenvolvimento ${ }^{21}$.

No tocante a CSC, também observa-se falhas dos profissionais quanto à informação ou explicação acerca da CSC. Em contrapartida, estudos realizados em Belo Horizonte e em Rondônia evidenciaram que mais da metade dos pais/cuidadores pesquisados mencionaram ter recebido informações acerca da $\operatorname{CSC}^{14,22}$.

Apesar da maioria dos participantes afirmarem que os profissionais mostram os dados que registram na CSC, uma parcela considerável dos cuidadores $(44,8 \%)$ nega essa ação profissional. Esse é um aspecto que também merece atenção, pois os marcos do desenvolvimento para a idade, as curvas do peso, da altura, do perímetro cefálico e do 
IMC, contidos na CSC, facilitam o entendimento dos pais sobre a situação da saúde da criança ${ }^{17}$. Logo, a Caderneta é um instrumento potente para aproximar os profissionais das famílias e facilitar a comunicação sobre o processo de crescimento e desenvolvimento infanti ${ }^{23}$.

Evidenciou-se associação estatística entre o envolvimento dos cuidadores na vigilância do desenvolvimento infantil na consulta à criança e a utilização da caderneta nesse processo, com as variáveis sociodemográficas e econômicas dos cuidadores. Igualmente, outros estudos relacionados à VDI também revelaram associação significativa entre essa e outras variáveis ${ }^{14,22}$.

A associação da renda com o envolvimento dos cuidadores na vigilância do desenvolvimento na consulta sugere que os núcleos familiares, que possuem renda menor que um salário mínimo, foram os menos questionados acerca do desenvolvimento de suas crianças. Nesse contexto, foi evidente a maior proporção da ausência de orientações de como estimular a criança no domicílio e a apresentação dos dados registrados na caderneta da criança aos familiares de baixa renda. Sobre isso, estudos internacionais apontam que grupos com renda familiar mais favorecida apresentam mais facilidade nas atividades de educação em saúde, como também de participação dos pais na estimulação do desenvolvimento e os cuidados com a criança em casa são maiores nesse grupo ${ }^{1}$.

Outrossim, o fato acima apresentado pode ocorrer também pelo fato dos profissionais associarem a pobreza a falta de cuidados e atenção com a criança no ambiente domiciliar, ou a questão cultural dos lares com pouco poder aquisitivo e que, por isso, deixaram de considerar e envolver essas famílias no que se refere ao desenvolvimento infantil de suas crianças e, consequentemente, não orientaram quanto à estimulação no ambiente doméstico e não mostram os registros realizados. O núcleo familiar em que se insere a criança percebe o mundo a partir de seus valores e das relações com o seu meio cultural. Essas inter-relações em seus contextos sociais acontecem desde o nascimento, e perpetuam-se no decorrer de suas vidas, interferindo diretamente na interação desses com as pessoas e os espaços em seus entornos ${ }^{24}$.

Ainda que os dados revelem associação entre a renda com a falta do envolvimento familiar na VDI, pesquisas nacionais e internacionais evidenciam o alto potencial da família na promoção e vigilância do desenvolvimento na primeira infância e que a baixa condição econômica pode ser superada quando os profissionais e os cuidadores estão engajados em ações para o pleno desenvolvimento infantil ${ }^{6,12,17 .}$

Com relação à quantidade de filhos, ocorreu significância estatística com as variáveis recebimento de alguma orientação de como estimular a criança em casa e se o profissional mostrou o registro dos dados na caderneta. Os dados evidenciam que cuidadores com apenas um filho receberam menos orientações do que aqueles que com dois filhos. Uma possível explicação para isso é que pais/cuidadores com dois filhos conhecem mais acerca do desenvolvimento infantil e tem mais experiência no cuidado, por isso, permanecem mais alertas ao desenvolvimento da criança, exigem a utilização da $\mathrm{CSC}^{25}$, questionando os profissionais sobre 
os registros realizados e o desenvolvimento da criança.

No entanto, em se tratando de mostrar o registro dos dados da CSC, os profissionais costumam apresentar também para os cuidadores com apenas um filho. Isso pode ser devido ao fato das primíparas, devido uma série de mudanças em suas vidas, com maior responsabilização, amadurecimento e se adaptando a cuidar de um bebê, estão sempre em busca de aprendizagem e, por isso, buscam muitas vezes os profissionais de saúde ${ }^{26}$.

\section{CONCLUSÃO}

A participação da família é de suma relevância para a promoção do desenvolvimento na primeira infância, no entanto, os achados evidenciam fragilidades nas ações dos profissionais quanto à Vigilância do Desenvolvimento Infantil, assim como, a família não é orientada para a promoção do desenvolvimento da criança. Isso porque, as práticas profissionais durante a consulta à criança não permitem a colaboração construtiva da família na caderneta de saúde das crianças, fato que dificulta a continuidade do seguimento da criança e estímulo ao pleno desenvolvimento no ambiente domiciliar, que deveria ser orientado e apoiado pela equipe profissional, especialmente na Estratégia Saúde da Família.

Enfatiza-se a importância da existência de vínculos positivos entre os cuidadores e os profissionais de saúde, para que suas práticas estejam em consonância com o contexto no qual a criança está inserida e, assim, favorecer a promoção do desenvolvimento saudável. Faz-se também necessário que os gestores proporcionem formação para os profissionais de saúde, para que o cuidado com a criança seja qualificado. Adicionalmente, sugere-se novos estudos que busquem evidenciar os motivos pelos quais os profissionais tem dificuldade de incluir a família em suas práticas de vigilância do desenvolvimento.

Esse estudo teve como limitações o fato da amostra ser selecionada por conveniência nas USFs de apenas um Distrito Sanitário do município, o que não permite um olhar mais ampliado dos cuidadores na VDI, além disso, algumas questões não foram respondidas pelos cuidadores, por falta de lembrança da prática profissional realizada.

\section{Agradecimentos}

Ao CNPq pelo Auxílio financeiro do Conselho Nacional de Desenvolvimento Científico e Tecnológico (Processo no 407850/2018-0) e pela bolsa de Iniciação Científica a uma das autoras. 


\section{REFERÊNCIAS}

1. Tran TD, Luchters S, Fisher J. Early childhood development: impact of national human development, family poverty, parenting practices and access to early childhood education. Child Care Health Dev. 2017; 43(3):415-426.

2. Reichert APS, Vieira DS, Santos NCCB, Albuquerque TM, Collet N, Vaz EMC. Vigilância do crescimento e desenvolvimento: análise dos registros na caderneta de saúde da criança. Cogitare Enferm. 2016; 21(4):01-09.

3. Chan M, Lake A, Hansen K. The early years: silent emergency or unique opportunity?. J Lancet. 2017; 389(10064):1113.

4. Black MM, Walker SP, Fernald LCH, Andersen CT, DiGirolamo AM, Lu C, et al. Early childhood development coming of age: science through the life course. J Lancet. 2017; 389(10064):77-90.

5. Silva ÂCDD, Engstron EM, Miranda CT. Fatores associados ao desenvolvimento neuropsicomotor em crianças de 6-18 meses de vida inseridas em creches públicas do Município de João Pessoa, Paraíba, Brasil. Cad. Saúde Pública. 2015; 31(9):1881-193.

6. Reichert APS, Collet N, Eickmann SH, Lima MC. Vigilância do desenvolvimento infantil: estudo de intervenção com enfermeiros da Estratégia Saúde da Família. Rev. Latinoam. Enferm. 2015; 23(5):954-962.

7. Ministério da Saúde, Secretaria de Atenção à Saúde, Departamento de Ações Programáticas Estratégicas. Política Nacional de Atenção Integral à Saúde da Criança: orientações para implementação. Brasília. Ministério da Saúde, 2018.

8. Figueiras AC, Souza ICN, Ríos VG, Benguigui Y. Manual para la vigilancia del desarrollo infantil (0-6 años) en el contexto de AIEPI. 2 ed. Washington, 2011.

9. Vieira DS, Santos NCCB, Costa DKG, Pereira MM, Vaz EMC, Reichert APS. Registro de ações para prevenção de morbidade infantil na caderneta de saúde da criança. Ciênc. Saúde coletiva (online). 2016; 21(7):2305-2313.

10. Silva FB, Gaíva MAM. Preenchimento da Caderneta de Saúde da Criança: percepção dos profissionais. Ciênc. Cuid. Saúde. 2015; 14(2):1027-1034.

11. Almeida AP, Ceballos LC, Barbosa ARC, Nogueira DA, Moreira DS. O registro do crescimento e desenvolvimento da criança na caderneta de saúde. Rev. Enferm. UERJ. 2017; 25:e16895.
12. Shah $R$, Isaia A, Schwartz A, Atkins M. Encouraging Parenting Behaviors That Promote Early Childhood Development Among Caregivers From Low-Income Urban Communities: A Randomized Static Group Comparison Trial of a Primary Care-Based Parenting Program. Matern Child Health J. 2019; 23(1):39-46.

13. Urke HB, Contreras M, Matanda DJ. The Influence of Maternal and Household Resources, and Parental Psychosocial Child Stimulation on Early Childhood Development: A Cross-Sectional Study of Children 36-59 Months in Honduras. Int J Environ Res Public Health. 2018; 15(5):926.

14. Amorim LP, Senna MIB, Gomes VE, Amaral JHLD, Vasconcelos M, Silva AG, et al. Preenchimento da Caderneta de Saúde da Criança nos serviços de saúde em Belo Horizonte, Minas Gerais, Brasil. Epidemiol. Serv. Saúde. 2018; 27(1):e201701116.

15. Gaiva MAM, Monteschio CAC, Moreira MDS, Salge AKM. Avaliação do crescimento e desenvolvimento infantil na consulta de enfermagem. Ar. Enferm. 2018; 36(1):9-21.

16. Franco LJ, Passos ADC. Fundamentos de epidemiologia. 2 ed. São Paulo: Editora Manole; 2011

17. Lima LG, Nobre CS, Lopes ACMU, Rolim KMC, Albuquerque CM, Araujo MAL. A Utilização da Caderneta de Saúde da Criança no acompanhamento Infantil. Rev. Bras. Ciênc. Saúde. 2016, 20(2):167-174.

18. Hirai AH, Kogan MD, Kandasamy V, Reuland C, Bethell C. Prevalence and variation of developmental screening and surveillance in early childhood. JAMA Pediatr. 2018; 172(9):857-866.

19. D'Aprano A, Silburn S, Johnston V, Bailie R, Mensah F, Oberklaid $F$, et al. Challenges in monitoring the development of young children in remote Aboriginal health services: clinical audit findings and recommendations for improving practice. Rural Remote Health. 2016; 13(3):3852

20. Ronfani L, Vecchi Brumatti L, Mariuz M, Tognin V, Bin M, Ferluga $\mathrm{V}$, et al. The complexinteraction between home environment, socioeconomic status, maternal IQ and early child neurocognitive development: a multivariate analysis of data collected in a newborn cohort study. PLoS ONE. 2015; 10(5): e0127052.

21. Venancio SI. Por que investir na primeira infância?. Rev. Latino-Am. Enfermagem Internet]. 2020 [citado 2020 Maio 26] ; 28: e3253.

22. Freitas JLG, Pereira PPS, Moreira KFA, Órfão NH, Cavalcante DF, Nascimento RC et al. Preenchimento da caderneta de saúde da criança na primeira infância. Rev. Bras. Promoç. Saúde. 2019; 32:8407. 
23. Rosolem LH, Contiero-Toninato AP, Sanguino GZ, Bonati PCR, Rezende VD, Mello DF et al. Caderneta de saúde da criança: coordenação do cuidado e acesso à saúde. Cogitare Enferm, 2019; 24:e61496.

24. Correa W, Minetto MF, Crepaldi MA. Família como Promotora do Desenvolvimento de Crianças que Apresentam Atrasos. Pensando Fam. 2018; 22(1):44-58.

25. Abud SM, Gaíva MAM. Registro dos dados de crescimento e desenvolvimento na caderneta de saúde da criança. Rev. Gaúcha Enferm. (Online). 2015; 36(2):97-105.
26. Zanatta E, Pereira CRR, Alves AP. A experiência da maternidade pela primeira vez: as mudanças vivenciadas no tornar-se mãe. Pesqui. Prát. Psicissociais. 2017; 12(3):16.

\section{CORRESPONDÊNCIA}

Iolanda Carlli da Silva Bezerra.

Rua Aurélio Guedes Cavalcante 718,

Camboinha II, Cabedelo.

E-mail: iolandacarlli@gamil.com. 